\title{
Heme oxygenase-1 in pregnancy and cancer: similarities in cellular invasion, cytoprotection, angiogenesis, and immunomodulation
}

\section{Hui Zhao*, Maide Ozen, Ronald J. Wong and David K. Stevenson}

Department of Pediatrics, Division of Neonatal and Developmental Medicine, Stanford University School of Medicine, Stanford, CA, USA

\section{Edited by:}

Ana Claudia Zenclussen,

Otto-von-Guericke University,

Germany

\section{Reviewed by:}

Anne Schumacher, Otto-von-Guericke University, Germany

Eric George, University of Mississippi Medical Center, USA

\section{*Correspondence:}

Hui Zhao, Department of Pediatrics,

Division of Neonatal and

Developmental Medicine, Stanford

University School of Medicine,

300 Pasteur Drive, Rm S230,

Stanford, CA 94305-5208, USA

e-mail: huizhao2@stanford.edu
Pregnancy can be defined as a "permissible" process, where a semi-allogeneic fetus and placenta are allowed to grow and survive within the mother. Similarly, in tumor growth, antigen-specific malignant cells proliferate and evade into normal tissues of the host. The microenvironments of the placenta and tumors are amazingly comparable, sharing similar mechanisms exploited by fetal or cancer cells with regard to surviving in a hypoxic microenvironment, invading tissues via degradation and vasculogenesis, and escaping host attack through immune privilege. Heme oxygease-1 (HO-1) is a stress-response protein that has antioxidative, anti-apoptotic, pro-angiogenic, and anti-inflammatory properties. Although a large volume of research has been published in recent years investigating the possible role(s) of HO-1 in pregnancy and in cancer development, the molecular mechanisms that regulate these "yin-yang" processes have still not been fully elucidated. Here, we summarize and compare pregnancy and cancer development, focusing primarily on the function of HO-1 in cellular invasion, cytoprotection, angiogenesis, and immunomodulation. Due to the similarities of both processes, a thorough understanding of the molecular mechanisms of each process may reveal and guide the development of new approaches to prevent not only pregnancy disorders; but also, to study cancer.

Keywords: Placenta, trophoblast invasion, angiogenesis, immunosuppression, tolerogenic dendritic cells (tDC), alternatively activated macrophage (M2)

\section{INTRODUCTION}

The development of the placenta is a highly regulated and complex physiologic process that is required to ensure proper fetal development, and the creation of life. In contrast, the formation of cancer is a pathologic process, characterized by uncontrolled growth of malignant cells that may ultimately lead to death. Even with these obvious differences, in some aspects, these two "yin-yang" processes actually are very similar, incorporating comparable mechanisms regulating cell growth, cell invasion, neovascularization, and immunotolerance (Holtan et al., 2009; Figure 1). In early placental development, trophoblast cells, originating from the developing embryo, implant in the uterine wall and encounter extremely harsh conditions, such as extreme hypoxia ( $\sim 1-2 \%$ oxygen), lack of a vasculature supply, and potential immune attacks from the maternal immune system. Interestingly, this environment is similar to that for an invading tumor. Malignant cells must also aggressively invade into normal tissue, establish a vasculature system, and defend against a host's immune response. Somehow, both trophoblast, and malignant cells are able to successfully survive and grow (Holtan et al., 2009).

Heme oxygenase $(\mathrm{HO})$ is a gene that is conserved across all biological kingdoms (animals, plants, and bacteria), and its role in heme degradation has been well understood. There are 3 known isoforms: the inducible $\mathrm{HO}-1$, the constitutively expressed HO-2, and the pseudogene HO-3 (Cruse and Maines, 1988; Maines, 1988, 1997; McCoubrey et al., 1997).
HO-1 can be upregulated by its substrate heme and also by heat shock, heavy metals, endotoxin, prostaglandins, inflammatory cytokines, etc. Therefore, it is referred to as a stressresponse protein. HO-1 is ubiquitously expressed, however, its expression level and the associated functions vary from cell type to cell type. $\mathrm{HO}$ and its metabolites, carbon monoxide (CO), iron, and bilirubin, also exhibit significant antioxidative, cytoprotective, pro-angiogenic, neurotransmitting, and antiinflammatory properties (Abraham et al., 1988; Marks et al., 1991; Choi and Alam, 1996). Its beneficial effects have been observed in atherosclerosis, diabetes, ischemia/reperfusion injury, as well as in organ transplantation (Shibahara et al., 2003; Exner et al., 2004).

The $\mathrm{HO} / \mathrm{CO}$ system is believed to be important in the maintenance of a healthy pregnancy. Several investigators have reported an association between a low expression of HO-1 with pregnancy disorders. For example, immunohistochemical staining of HO-1 in human placentas was lower in pre-eclamptic patients compared to pregnant controls (Ahmed et al., 2000). End-tidal breath CO levels were lower in hypertensive pregnant women, especially those with severe pre-eclampsia, than pregnant healthy women (Baum et al., 2000; Kreiser et al., 2004). Reduced HO-1 expression was also found in mouse models of spontaneous abortions (Zenclussen et al., 2002, 2006b).

Similarly, HO-1 has been linked with tumor induction, growth, and metastasis. Induction of HO-1 expression has been observed 


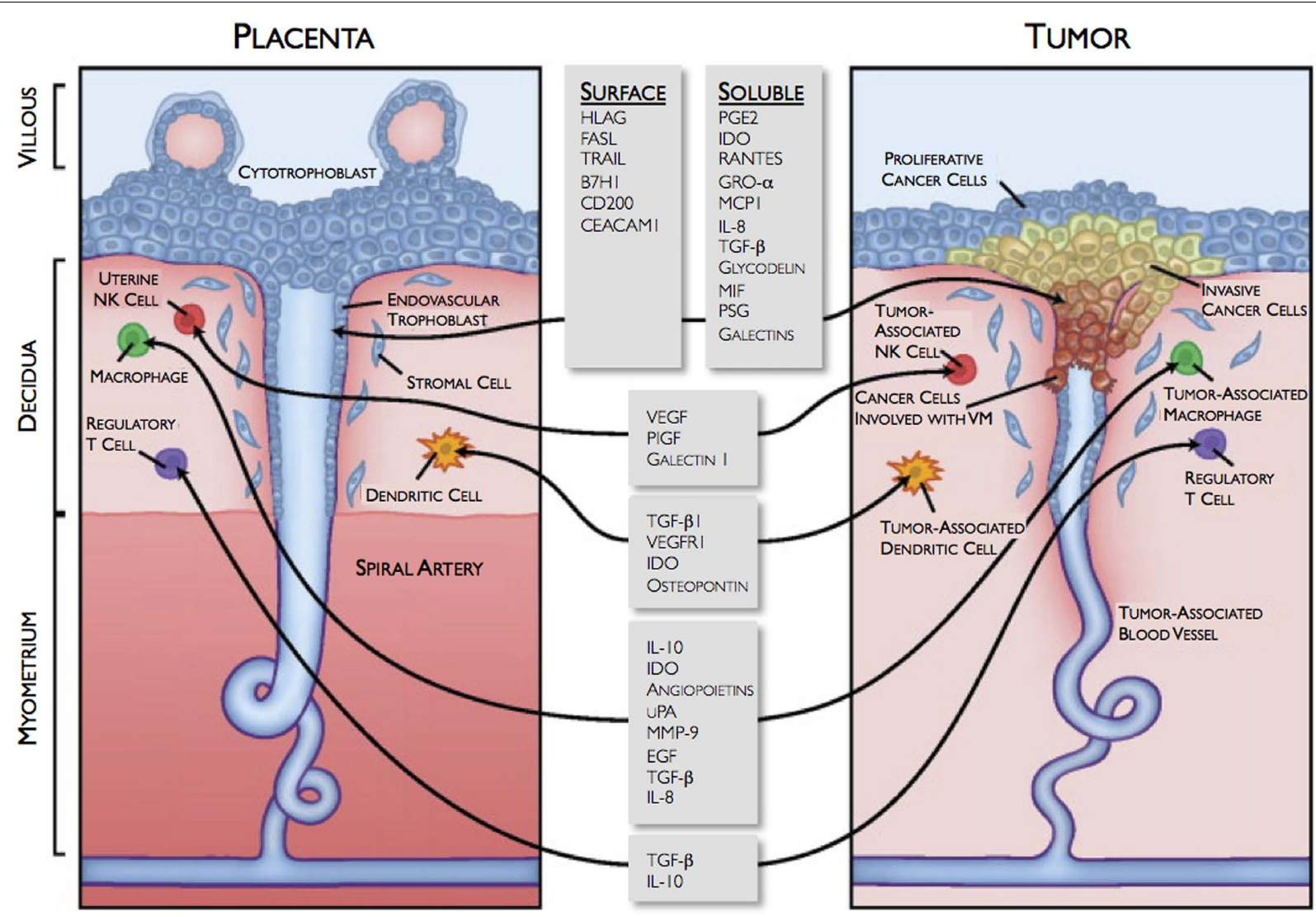

FIGURE 1 | Similarities between the fetomaternal interface and tumor microenvironment. VM, vasculogenic mimicry. Reprinted with permission from Holtan etal. (2009) (C2009 Mayo Foundation for Medical Education and Research.

in tumors, such as lymphosarcomas, prostatic cancers, glioblastomas, hepatomas, melanomas, pancreatic cancers, and chronic myeloid leukemias (Was et al., 2010). Tumors exhibit strong neovascularization and massive hemorrhaging, and therefore can release significant amounts of heme, which can then directly induce HO-1 expression and confer cytoprotection from oxidative injury. HO-1 is also involved in tumor angiogenesis and stimulating tumor-associated macrophages (TAMs; Was et al., 2006). Therefore, HO-1 may be involved in tumor survival and progression.

This review summarizes the common properties shared by the processes of placental and cancer development, emphasizing the role of HO-1 on cytoprotection, angiogenesis, and immune privilege. Mechanisms used by malignant cells to proliferate, invade, and immunosuppress may also apply to those used by trophoblast cells in the development of the fetomaternal interface. The understanding of these two clinically different, yet biologically similar, processes may lead us to develop new approaches to prevent pregnancy disorders related to placental disorders.

\section{HO-1 EXPRESSION AND INVASIVENESS OF TROPHOBLAST AND TUMOR CELLS}

HO-1 expression has been studied in both human and mouse placentas. In human placental tissue, the results have been inconsistent (Lyall et al., 2000; McLean et al., 2000; Yoshiki et al., 2000; McLaughlin etal., 2003; Bilban et al., 2009), very likely due to the intra- and inter-tissue heterogeneity and different gestational ages studied. Due to the difficulties of human sample collection, most studies were performed using either term or preterm-delivered placentas. In addition, for most cases, the tissue collected was only from the placenta and without the placental bed, and used only for immunohistochemical studies.

To date, the most systematic study is from Lyall et al. (2000), who collected both the placenta and placental bed tissues from biopsies done in first two trimesters ( 8 and 19 weeks) as well as at term using a transcervical sampling technique. They found that in villous tissue (placenta), HO-1 was low and was not gestational age-dependent, while HO-2 displayed a spatial and temporal pattern of expression: $\mathrm{HO}-2$ was predominant in syncytiotrophoblast in the first trimester and decreased at term, while endothelial immunostaining was weak in the first trimester, but increased by term. However, within the placental bed, both HO-1 and HO-2 were intensely expressed in extravillous trophoblasts (EVTs, with high invasiveness), but absent from the proximal cytotrophoblast (CTBs, with less invasiveness) layers of cell column (Lyall et al., 2000). EVTs are highly proliferative and invasive in nature. They are involved in the attachment of the placenta to the decidua (uterine wall) by migrating through the syncytiotrophoblast and 
also in the modification of the spiral arteries. The authors believe that since HO-1 and HO-2 is highly expressed in EVTs, HO plays a role in trophoblast invasion and transformation of spiral arteries.

However, Bilban et al. (2009) also collected first trimester human placental tissues and compared the mRNA expression profiles of EVTs to the less invasive CTBs. They found that HO-1 was actually downregulated in EVTs. In addition, a high expression of HO-1 in the proliferative, Ki67-positive cell column was detected, in contrast to the low levels observed in non-cycling, Ki67-negative, invasive EVTs. They further showed that HO-1 can negatively regulate the motility of trophoblasts by acting via the nuclear hormone peroxisome proliferator-activated receptor (PPAR) $-\gamma$. The contradictory results from the two groups may be due to the time of placental bed sample collection. During the first 10-12 weeks of gestation, EVTs form "plugs" that can prevent maternal blood flow into the intervillous space and creating a hypoxic environment. During their migration away from the villi, EVTs differentiate into an invasive phenotype. Dynamic changes of oxygen level, availability of heme, and variations in tissue sampling may affect downstream results and account for the conflicting findings. Despite this, $\mathrm{HO}-1$ is still believed to be critically involved in mediating the differentiation, invasion, and motility of EVTs during the first trimester of pregnancy.

Unlike that of the human, invasive trophoblasts in the mouse placenta have very shallow invasion into the decidua. Therefore, it is understandable that there is a weaker staining of HO-1 in the mouse decidua, which is mostly confined to the spongiotrophoblast layer in the junction zone, which serves as the interface between the maternal and embryonic sides of the placenta (Watanabe etal., 2004; Zhao etal., 2009). It is not clear if invasive trophoblasts or functional equivalent cells of human EVTs are located in the junction zone in the mouse placenta. Using histological examination as well as 3-D images from casted placentas, Zhao et al. (2009, 2011), Wong et al. (2012) observed that in HO1 heterozygote (Het, $\mathrm{HO}-1^{+/-}$) placentas, there is an increase in apoptosis that leads to markedly thinner junction zones compared to those in wild-type (Wt) placentas. Therefore, in the mouse placenta, cells in the junction zone may be interesting candidates for investigating the function of HO-1 in decidual formation and spiral artery remodeling.

Interestingly, invasive trophoblast cells are strikingly similar to cancer cells in their capacities to proliferate, migrate, and die, making comparisons to cancer development very compelling. Both trophoblasts and cancer cells are rapidly dividing cells, where HO1 is abundantly expressed. Many human tumors produce $\mathrm{HO}-1$ and its expression is usually higher in cancer cells than in surrounding healthy tissues (Was et al., 2010). HO-1 is localized in either tumor cells or macrophages or both, but its exact location in transformed tissues depends on the type of tumor as well as its stage of development. For example, in human melanomas (TorisuItakura et al., 2000) or gliomas (Deininger et al., 2000), HO-1 was almost exclusively expressed in macrophages, which accumulated around the necrotic area. In human pancreatic carcinomas, $\mathrm{HO}-1$ immunoreactivity was found in both cancer and immune cells (Berberat et al., 2005). In rat hepatomas, HO-1 was found only in tumor cells (Doi et al., 1999).
In addition, a high expression of HO-1 may be associated with poor prognosis in patients with non-small cell lung cancers (Tsai et al., 2012). In contrast, patients with a high expression of HO-1 and colorectal cancers have a favorable prognosis, and those with oral squamous cell carcinomas have a low risk of lymph node metastases (Noh et al., 2013). The association of HO-1 and tumor cell invasion is also inconsistent and appears to vary among tumor types. For example, HO-1 inhibits invasion of breast cancer by suppressing the expression of matrix metalloproteinase-9 (MMP9; Lin et al., 2008), but promotes gastric cancer invasion via the sonic hedgehop signaling pathway (Xu et al., 2012). Therefore, the role of HO-1 in human malignant tumor growth may vary depending upon tumor type.

\section{EFFECTS OF HO-1 ON CYTOPROTECTION IN THE HYPOXIC ENVIRONMENT}

HO-1 is a well-known strong antioxidant that has been shown to protect various cell types from oxidative damage and to reduce the rate of apoptosis. The most convincing data describing these cytoprotective properties are from studies from Poss and Tonegawa (1997), who first established the HO-1 knockout $\left(\mathrm{HO}-1^{-/-}\right)$mouse. Using this mouse model, they showed that HO-1 deficiency leads to severe oxidative stress with elevated lipid peroxidation, cardiovascular damage, and progressive chronic inflammation in the kidney and liver. Moreover, fibroblasts isolated from $\mathrm{HO}-1^{-/}$mice showed increased production of reactive oxygen species (ROS) and reduced cell viability when exposed to various oxidants (Poss and Tonegawa, 1997).

In early pregnancy, the fetal environment is extremely "hypoxic." From 8 to 10 weeks of gestation, Oxygen $\left(\mathrm{O}_{2}\right)$ levels in the intervillous space is around $18 \mathrm{~mm} \mathrm{Hg}$ compared to 40 and $61 \mathrm{~mm} \mathrm{Hg}$ in the endometrium and at the end of the third trimester, respectively. From 12 to 13 weeks of gestation, placental $\mathrm{O}_{2}$ levels increase to levels similar to those measured in the endometrium (Rodesch et al., 1992; Jaffe et al., 1997; Burton et al., 1999). In first trimester, an hypoxic environment may be important in the regulation of trophoblast differentiation that is mediated through a complex set of interactions between factors associated with oxidative stress, oxygen-sensing, and the release of inflammatory cytokine (James et al., 2006). It is currently unclear whether this increase of ROS production in trophoblasts is a direct result of hypoxia as these cells have the ability to control oxidative stress using several different pathways. Genes responsive to hypoxia are those that enhance $\mathrm{O}_{2}$ delivery, decrease $\mathrm{O}_{2}$ consumption, or regulate cellular metabolism.

The response of HO-1 to hypoxia or its association with the trophoblast differentiation has not been well established. On one hand, as $\mathrm{O}_{2}$ availability is reduced, $\mathrm{HO}$ activity actually decreases since molecular $\mathrm{O}_{2}$ is a co-factor required for heme breakdown (Appleton et al., 2002). On the other hand, although HO-1 mRNA and protein levels have been shown to increase in response to hypoxia in several organ systems, placental HO-1 expression under hypoxia is controversial. Using human term placental explants, Appleton et al. (2003) found that HO-1 levels were unaffected when the explants were exposed to different $\mathrm{O}_{2}$ tensions. However, George et al. (2012) have reported that 
48 h of chronic hypoxic exposure down-regulated HO-1 expression in cultured rat placental villous explants. In contrast, in an established ischemia [reduced uterine perfusion pressure (RUPP]) model, they found that placental HO-1 increased compared to non-RUPP-treated controls (George et al., 2011b). Interestingly, using a rat trophoblast stem cell line, Zenclussen et al. (2011) found that HO-1 inhibition could impair cell viability and abolish their differentiation to giant cells.

During tumor proliferation, regions of the tumor may have significantly lower $\mathrm{O}_{2}$ concentrations than healthy tissues as they rapidly outgrows their own blood supplies. In order to support continuous growth and proliferation under these hypoxic conditions, cancer cells can alter their metabolism as well as increase their cytoprotective enzymes, such as HO-1 and its metabolite CO. Pharmacological or genetic activation of HO-1 significantly improves survival of many tumors, such as hepatomas, thyroid carcinomas, chronic myeloid leukemias, gastric carcinomas, and gliomas. In contrast, HO-1 inhibition can reduce colon carcinomas, acute myeloid leukemias, and hormone-refractory prostate cancers (Was et al., 2010). Expression of HO-1 is further elevated in response to anti-cancer treatments, such as chemotherapy, radiotherapy, and photodynamic therapy, all of which can induce hypoxia, oxidation, and pro-apoptosis or pro-necrosis in cancer cells (Was et al., 2010). Induction of HO-1 seems to counteract these treatments, hindering the effectiveness of anti-cancer therapies, and inducing resistance.

The mechanisms involved in the cytoprotective effects of $\mathrm{HO}-1$ in tumor cells are still not fully known. One pathway that has been postulated is the removal of free heme. Another is the increase of cellular biliverdin/bilirubin, both strong antioxidants. In tumors, there are high free heme levels due to massive hemorrhaging and necrosis of host cells and tissues. In the fetomaternal interface, cell apoptosis is constantly occurring, but it is not clear if free heme resulting from cell death has any role in affecting $\mathrm{HO}-1$ expression.

\section{H0-1 IN PLACENTAL VASCULATURE DEVELOPMENT}

Normal placental development is a balance of angiogenesis and vasculogenesis, which is believed to be mediated by a crosstalk between different cell types. Although HO-1-deficient animals do not show any visible phenotype suggestive of vasculature defects, accumulating evidence reveals an association between HO-1 deficiency and poor angiogenesis. Direct effects of HO- 1 on angiogenesis have been mostly studied in endothelial or endothelial progenitor cells (EPCs). Deramaudt et al. (1998) have shown that the overexpression of HO-1 enhances endothelial cell proliferation. Li Volti et al. (2005) showed that by inhibiting HO-1 by antisense strategies, both endothelial cell proliferation and capillary formation decrease in vitro, which may be associated with the cell cycle. However, the effect of HO-1 on angiogenesis can also be indirect and mediated through pro-angiogenic factors or the recruitment of EPCs. The upregulation of HO-1 (and hence $\mathrm{CO}$ ) increases the synthesis of the pro-angiogenic factors, vascular endothelial growth factor (VEGF), monocyte chemotactic protein 1 (MCP-1 or CCL2), transforming growth factor (TGF)- $\beta$, and IL-8; and decreases production of anti-angiogenic mediators: soluble Flt-1 (sFlt-1), soluble endoglin (sEng), and CXCL10 (Cudmore et al., 2007; Deshane et al., 2007; Dulak et al., 2008;
Loboda et al., 2008), to result in a stimulation of angiogenesis and vasculogenesis.

Stromal cell-derived factor-1 (SDF-1 or CXCL12) plays a major regulatory role in the migration, recruitment, and retention of EPCs to areas of ischemic injury and contributes to neovascularization. Inactivation of SDF-1 or its receptor in mice leads to intrauterine deaths due to abnormalities in vascular development (Ma etal., 1998; Ratajczak etal., 2006). The first study revealing that HO-1 is directly involved in regulating angiogenesis via the SDF-1 pathway is from the laboratory of Agarwal (Deshane et al., 2007). They demonstrated that SDF-1 upregulates HO-1 in endothelial cells through a protein kinase C-dependent, but VEGFindependent, pathway. In the absence of $\mathrm{HO}-1, \mathrm{SDF}-1$ was unable to promote endothelial tube formation and migration or induce the formation of capillary sprouts and aortic rings. Interestingly, these defects can be reversed by exogenous $\mathrm{CO}$ administration. In addition, they observed that the impairment of wound healing in HO-1-deficient mice is due, in part, to a reduction of EPC recruitment and capillary formation. These HO-1-deficient EPCs were unable to re-endothelialize the retina after ischemic injury (Deshane et al., 2007).

In the placenta, SDF-1 expression has been found in trophoblast cells, especially EVTs. Hanna et al. (2003) showed that SDF-1 produced by EVTs induces the specific migration of human blood $\mathrm{CD}_{16}{ }^{-}$natural killer $(\mathrm{NK})$ cells via CXCR-4 receptors. $\mathrm{CD} 16^{-} \mathrm{NK}$ cells are a unique subset of blood NK cells that share a similar phenotype with decidual NK ( $\mathrm{dNK}$ ) cells. Therefore, these recruited CD16 ${ }^{-}$NK cells may be the actual precursors of $\mathrm{dNK}$ cells, which play important roles in spiral artery remodeling and placental vasculature formation. To date, it is unknown if $\mathrm{HO}-1$ is involved in uterine NK ( $\mathrm{uNK}$ ) recruitment by SDF-1. Since significant reductions in uNK cells and in uNK-related angiogenic factors were indeed found in the HO-1-deficient (Het, HO- $1^{+/-}$) placenta (Zenclussen et al., 2011; Zhao et al., 2011; Linzke et al., 2014), we speculate that HO-1 may have a regulatory role in mediating the crosstalk between EVTs and decidual stromal cells, including uNK cells (Du et al., 2012; Ren et al., 2012).

Expression of HO-1 in endothelial cells has been shown to also promote angiogenesis by downregulating anti-angiogenic mediators. Cudmore et al. (2007) demonstrated that adenoviral overexpression of HO-1 diminishes the production of anti-angiogenic sFlt1 receptor and sEng in response to the VEGF ligand in endothelial cells. In contrast, significantly higher levels of sFlt1 and sEng were found in HO-1-deficient compared to Wt mice. George et al. (2012) showed that HO-1 reduces hypoxia-induced sFlt-1 levels and oxidative stress in placental villi through $\mathrm{CO}$ and bilirubin. Since sFlt1 and sEng are key mediators in the pathogenesis of preeclampsia, the observation that $\mathrm{HO}-1$ can suppress the release of sFlt1 and sEng was further investigated. Zhao et al. (2009) found that maternal plasma sFlt-1 levels, as well as diastolic blood pressures, were significantly elevated in pregnant HO-1 Het mice compared to Wt mice, suggesting that pregnant HO-1-deficient dams have characteristics similar to those in pre-eclampsia. Moreover, George et al. (2011a) also showed that an induction of HO-1 could alleviate sFlt-1-induced hypertension in pregnant rats.

The placenta has a complex and well-organized vascular network comprised of both maternal and fetal vessels. In early 
pregnancy, the uterine spiral arteries become remodeled into low resistance and high capacitance vessels, which is necessary to support the dynamically growing placenta and fetus. The association of HO-1 and uteroplacental vascular formation has been investigated by Zhao et al. (2011), Wong et al. (2012) using HO-1 Het mice. They collected placentas at E10.5, since this gestational age corresponds to the second trimester in human pregnancies, when the uteroplacental vasculature is becoming established. Histochemical staining revealed significant differences in the placental vasculature from HO-1 Het dams compared to pregnant Wt dams, such as thinner junction zones, a reduction in maternal sinusoidal number and size in the labyrinth, and a disorganization of fetal capillaries. These observations suggest that HO-1 deficiency is associated with vascular malformations in the labyrinth (Zhao et al., 2011). A vascular corrosion technique to cast placentas and $\mathrm{CT}$ imaging to reconstruct $3 \mathrm{D}$ images were used to visualize the vasculature of the placentas at $\sim$ E16.5. They found that HO-1 Het placentas were smaller with a reduced labyrinth vessel volume and a thin maternal vascular region. Spiral arteries also appeared to be highly branched, but found to have smaller diameters (Zhao et al., 2011; Wong et al., 2012). They concluded that a partial deficiency of HO-1 leads to insufficient spiral artery remodeling and enlargement, which may by an underlying cause of pregnancy disorders, such as pre-eclampsia and intrauterine growth restriction (IUGR).

The formation of the uteroplacental vasculature network is a very complex event that involves not only endothelial cells, but also, trophoblast, uNK, decidual stromal, and infiltrating immune cells. These cells produce many cytokines, chemokines, growth factors, and angiogenic factors. Zhao et al. (2011) used PCR arrays to measure and then compare gene expression profiles from pregnant Wt and HO-1 Het uteri. Genes encoding for pro-angiogenic factors, such as growth factors, matrix metallopeptidase, and cytokines/chemokines, were significantly downregulated, while genes controlling anti-angiogenic factors were upregulated in $\mathrm{HO}$ 1 Het uteri. These data strongly suggest that the role of HO-1 in placental vasculature development is not due to a single event, but involves several cell types and the regulation of complex series of multiple steps.

Tumor angiogenesis initially begins with cancer cells releasing signaling molecules to the surrounding host tissues. These signals activate specific host genes that, in turn, stimulate the synthesis of proteins that stimulate new blood vessel growth. Unlike the tightly regulated and organized vasculature of the placenta, blood vessels of tumors are highly aberrant, dysfunctional, and uncontrolled. Despite the differences, the basic mechanism of tumor angiogenesis is very similar to those of uteroplacental vascular formation. HO-1 has also been suggested to be pro-angiogenic in many different tumor models, such as melanomas, pancreatic cancers, lung carcinomas, and gliomas (Was et al., 2010). By either inhibiting or inducing $\mathrm{HO}-1$, researchers have found that $\mathrm{HO}-1$ plays a critical role in mediating both VEGF-stimulated endothelial cell proliferation and SDF-1-induced EPC recruitment (Was et al., 2010).

Like that occurring in the placenta, the vascularization of tumors is also a complex process, relying not only on endothelial cells, but also on infiltrating immune cells, such as macrophages. In vivo studies have showed that an increased expression of $\mathrm{HO}-1$ is associated with an augmented angiogenesis in human gliomas and melanomas. Mouse studies have shown that TAMs promote angiogenesis in tumors by both producing pro-angiogenic factors and physically assisting the sprouting of blood vessels. Further studies have shown that TAMs are comprised of phenotypically and functionally distinct subsets, including the pro-angiogenic $\left(\mathrm{TIE}^{+}{ }^{+}\right)$ and the angiostatic/inflammatory $\left(\mathrm{CD}_{11} \mathrm{c}^{+}\right)$macrophages. The location and ratio of these two populations vary by the type and grade of the tumor (Welford et al., 2011). Since HO-1 is highly expressed in macrophages, it would be interesting to understand how HO-1 contributes to the differentiation and function of TAM subsets. In addition, macrophages are also observed in both deciduae and labyrinths. It may be of further interest to characterize whether macrophages in the placenta are pro-angiogenic or angiostatic, and to investigate their function in uteroplacental vascular formation.

\section{H0-1 IMMUNOMODULATION AND FACILITATION OF IMMUNOTOLERANCE AND IMMUNE PRIVILEGE}

During early pregnancy, fetal-derived trophoblast cells, especially in EVTs, penetrate deep into the uterine wall to form the decidua. At the same time, many maternal leukocytes, including monocytes, macrophages, dendritic cells (DCs), NK cells, neutrophils, and regulatory $\mathrm{T}$ cells (Tregs), also infiltrate into the decidua in order to support early placental development. Interestingly, direct contact of the semi-allogeneic EVTs with maternal leukocytes and decidual stromal cells does not induce rejection, but instead results in immune privilege. Initial studies suggest that prevention of fetal rejection is associated with an increased ratio of $\mathrm{T}$ helper type 2 (Th2) compared to Th1 cytokines produced by maternal leukocytes. However, a wealth of follow-up studies indicates that tolerance mechanisms initiated here are much more complicated than initially thought. It appears to involve the adaption of both innate and adaptive immune responses, locally, and systemically, aided by endocrine pathways (Arck and Hecher, 2013; Erlebacher, 2013).

HO-1 has been long accepted as an anti-inflammatory and immunosuppressive molecule. Its induction by pharmacological activators, through gene transfer, and delivery of heme metabolites (CO and bilirubin) produce immunosuppressive effects in a variety of conditions or disorders, such as autoimmune disease and organ transplantation (Becker et al., 2007; Tzima et al., 2009). HO-1 is expressed in circulating monocytes and tissue macrophages. In HO-1 knockout mice, residential macrophages such as spleen sinusoidal lining cells and liver Kupffer cells, are absent (Kovtunovych et al., 2010); while adoptively transferring Wt macrophages to HO-1-deficient mice has been shown to reverse disease (Kovtunovych et al., 2014), suggesting an important role of HO-1 in macrophage survival and function. Lee and Chau (2002) reported that HO-1 in macrophages mediates the antiinflammatory function of IL-10, a classic Th2 cytokine. Inhibition of $\mathrm{HO}-1$ protein or $\mathrm{HO}$ activity significantly reverses the inhibitory effect of IL-10 on the production of tumor necrosis factor-alpha (TNF- $\alpha$ ) induced by lipopolysaccharide (LPS; Lee and Chau, 2002).

The adverse effects of HO- 1 deficiency related to immune regulation during pregnancy are mostly observed in the context of 
human and murine spontaneous abortions. It has been reported that women with early miscarriages are prone to have longer (GT)n repeats in the HO-1 promoter region, and thus a lower expression of HO-1 (Denschlag et al., 2004). Zenclussen et al. (2002) has used two different models to elucidate the importance of $\mathrm{HO}-1$ in spontaneous abortions. HO-1 levels in placentas and deciduae were reduced in mice with sonic stress or IL-12-induced abortions. When HO-1 was delivered to spontaneous abortion mice via adenoviral transfer, rates of abortion declined with an increase of the IL-4 to interferon-gamma (IFN- $\gamma$ ) ratio in spleen lymphocytes and a decrease in apoptosis at the fetomaternal interface (Zenclussen et al., 2006b). Similarly, induction of HO activity can increase the expression of Bag-1, an anti-apoptotic factor, at the fetomaternal interface as well as activate Tregs, to collectively result in a lower spontaneous abortion rate (Zenclussen et al., 2005). Inhibition of $\mathrm{HO}$ activity by treatment with zinc protoporphyrin $(\mathrm{ZnPP})$ not only increased abortion rates in normal as well as abortion-prone pregnancies; but also, was found to abolish the protective effects of the transfer of Tregs (Schumacher et al., 2012). In addition, transfer of Tregs from healthy pregnant mice into abortion-prone mice reduced fetal rejection rates and resulted in the upregulation of $\mathrm{HO}-1$ and TGF- $\beta$ at the fetomaternal interface (Zenclussen et al., 2006a). Even with so much evidence showing the importance of HO-1 in the maintenance of a normal pregnancy, the exact mechanisms responsible for immunoregulation in the fetomaternal interface have not been fully elucidated.

Decidual macrophages are present throughout gestation and reside near the cytotrophoblast shell in close contact with EVTs. Decidual macrophages phagocytose apoptotic EVTs and secrete IL-10 and indoleamine 2,3-dioxygenase (IDO), adding to the tolerogenic Th2 environment. Gene expression profiling showed that decidual macrophages are immunosuppressive and anti-inflammatory with high expression of CCL18,
CD206, insulin-like growth factor (IGF-1), IDO, and other genes associated with M2-polarized macrophages (Gustafsson et al., 2008). Recent evidence suggests that HO-1 is critically involved in macrophage polarization to the M2 phenotype (Weis etal., 2009; Choi etal., 2010; Sierra-Filardi et al., 2010; Figure 2). HO-1 expression levels are significantly higher in M2 macrophages and induction of $\mathrm{HO}-1$ can significantly enhance IL-10 production, while it has no effect on M1 cytokine production (Sierra-Filardi et al., 2010). More interestingly, HO-1 may mediate the M1 to M2 phenotypic switch when mice are treated with heme (Choi et al., 2010). Further investigations are needed to best understand the function of HO-1 in decidual macrophages and how HO-1 contributes to placental development.

Dendritic cells have also been found in mouse and human deciduae, and undergo a restricted $\mathrm{T}$ cell response to a fetal specific-antigen. Most decidual DCs (dDCs) remain in an immature and tolerogenic state (tDCs). These cells exhibit an altered capacity for antigen presentation, with reduced expression of costimulatory molecules and IL-12, but an enhanced production of IL-10. tDCs can promote immunotolerance by inducing effector $\mathrm{T}$ cell apoptosis and expansion of $\mathrm{CD} 4{ }^{+}$Tregs. In addition, even in syngeneic mouse pregnancies, depletion of uterine DCs causes failure of decidualization and resorption of embryos, suggesting that a potentially more complex role for DCs in the development of the fetomaternal interface other than just antigen presentation and secretion of immunosuppressive cytokines. Moreover, HO-1 contributes to the maintenance of immature and anti-inflammatory state tDCs. Chauveau et al. (2005) have shown that HO-1 is only expressed in immature tDCs, but not in mature DCs. HO-1 induction in mature DCs lead to a loss of DC immunogenicity and pro-inflammatory function. Moreau et al. (2009) reported that HO- 1 was required for $\mathrm{tDCs}$ to delay cardiac allograft rejection

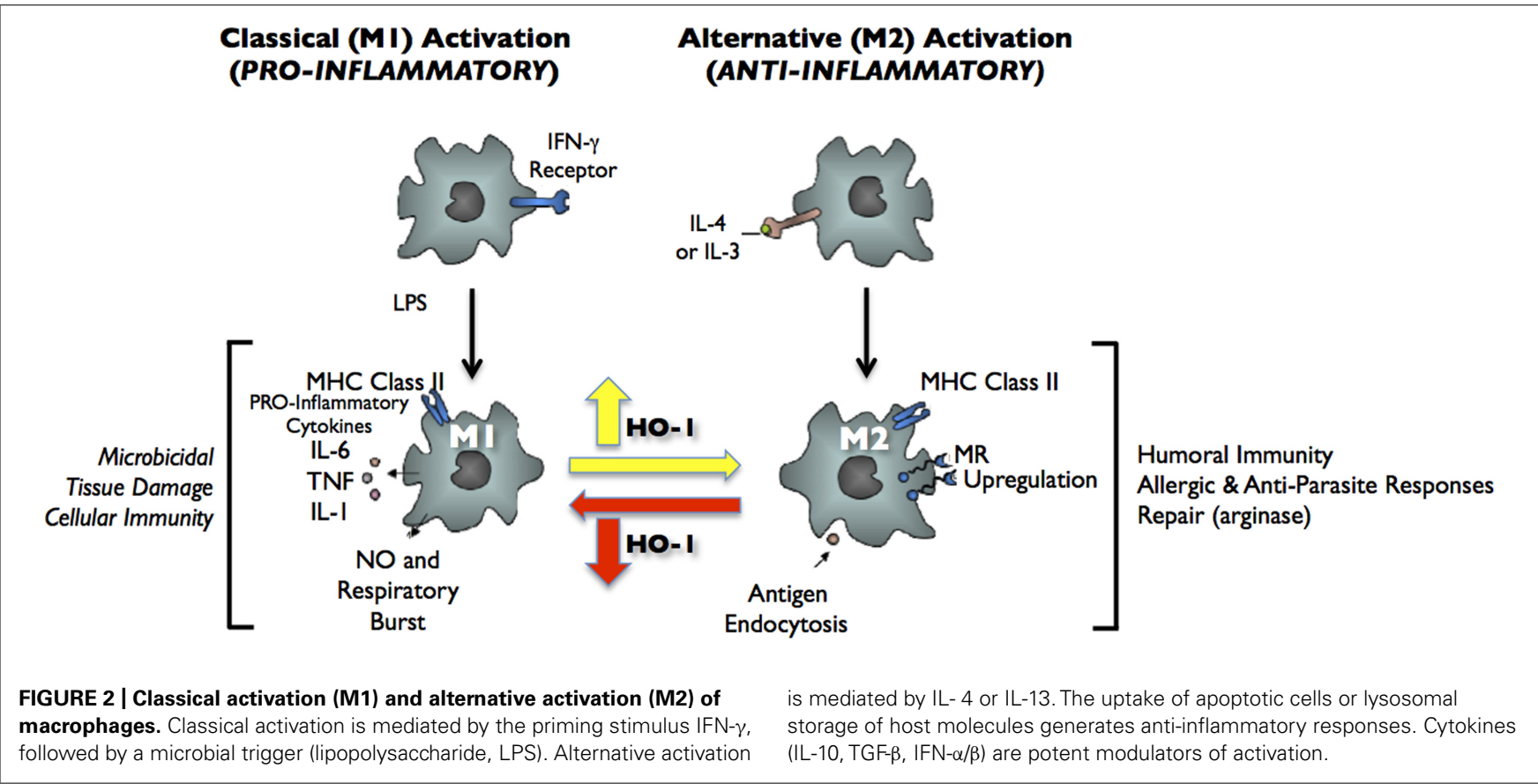


in the rat. Park et al. (2010) showed that expression of HO-1 by DCs is necessary for CD4 ${ }^{+}$Tregs to exert their immunoregulatory activity. Although DCs are well studied in pregnant uteri, the roles of HO-1 in the differentiation and function of dDCs are still not yet fully understood.

Tregs $\left(\mathrm{CD} 4^{+} \mathrm{CD} 25^{+}\right.$foxp $\left.3^{+}\right)$contribute to the implantation and maintenance of early pregnancy via their immunosuppressive and tolerance properties. Many studies have reported that the frequency of Tregs in mice and human deciduae increases during healthy pregnancies, while they decrease in abortion-prone pregnancies (Erlebacher, 2013). Rowe et al. (2012) found that in murine pregnancies the number of Tregs double, while Tregs specific to fetal antigens expand more than 100-fold. Although HO-1 is present in Tregs, the actual function of HO-1 in Tregs has been controversial. One study reported that the regulatory function of Tregs is lost when $\mathrm{HO}$ activity was abolished by a pharmacological inhibitor (Andersen et al., 2009), while another study demonstrated that an amplification of the Treg population following the same treatment (Biburger et al., 2010). Studies from HO- $1^{-/-}$mice have provided a definitive answer: HO-1 expression in Tregs is not required for Treg immunosuppression (Zelenay et al., 2007); instead, Treg function is likely to be affected indirectly by $\mathrm{HO}-1$ expression in antigen-presenting cells (APCs), such as DCs or macrophages (George et al., 2008). Indeed, Schumacher et al. (2012) found that HO-1 indirectly contributes to the expansion of the peripheral Treg population by maintaining maternal DCs in an immature state.

Similar to trophoblasts in pregnancy, malignant cells can express antigens that mediate recognition by host $\mathrm{CD} 8^{+} \mathrm{T}$ cells. These antigens are mostly the result of a point mutation in normal genes. Cancer cells may have hundreds or even thousands of mutations in their coding exons, contributing a large repertoire of antigens that can serve as potential immune targets. Despite an abundance of antigens, most cancers can still progress and evade attack by the immune system. This phenomenon of "tumor escape" may be due to immunosuppression and tolerance in the tumor microenvironment.

Most solid tumors consist of many types of infiltrating leukocytes, including macrophages, DCs, neutrophils, NK cells, and $\mathrm{T}$ and $\mathrm{B}$ cells. Interestingly, very similar to the immune microenvironment in the fetomaternal interface, these infiltrating cells are mostly polarized toward immunosuppressive and tumor-promoting phenotypes. For example, TAMs are the most abundant population and display an M2-like phenotype. They promote tumor angiogenesis, cell invasion and metastasis, and facilitate cytoprotection from chemotherapy-induced apoptosis. They exhibit an ineffective ability to present antigens, but can attract Tregs to inhibit T cell activation. Similarly, DCs in tumors are also immature and functionally incompetent with a tolerance phenotype. Cancer cells secrete substances, such as VEGF, TGF- $\beta$, hepatocyte growth factor, and osteopontin, that can suppress the maturation of DCs (Holtan et al., 2009). These immature, tDCs express high levels of the pro-angiogenic cytokine VEGF, the pro-inflammatory cytokines IL-6 and IL-8, the immunosuppressive mediators IL-10, cyclooxygenase 2 (COX2), TGF- $\beta$, and IDO. Besides macrophages and DCs, immature neutrophils, also called myeloid-derived suppressor cells (MDSCs), display suppressive functions of $\mathrm{T}$ and NK cells in the tumor site (Gabrilovich and Nagaraj, 2009).

Arnold etal. (2014) demonstrated tumor immune suppression by macrophages expressing high levels of HO-1. However, the mechanisms of $\mathrm{HO}-1$ on immunotolerance employed by macrophages, DCs, and MDSCs have not been fully elucidated. One possibility is through CO, a metabolite of heme degradation. It has been shown that CO promotes the development of tDCs (Remy et al., 2009) and inhibits T cell proliferation (Song et al., 2004). In addition, Zenclussen et al. (2011) and Linzke et al. (2014) have reported that CO inhalation successfully restores the Wt phenotype in HO-1 Het pregnancies. Another putative mechanism of HO-1 may be mediated through other heme binding enzymes, such as IDO (Curti et al., 2009) and NADPH oxidase (Ryter et al., 2006). IDO, a heme binding and tryptophan catabolic enzyme, has been suggested to be the key player involved in the inhibition of cell proliferation and induction of immunotolerance during infection, pregnancy, transplantation, autoimmunity, and hematologic malignancies (Curti et al., 2009). Jung et al. (2010) found that murine DC maturation depends on IDO expression via a HO-1-dependent pathway. They found that in IDO deficiency, there is a loss of maturation of DCs in vitro and in vivo. In addition, inhibition of $\mathrm{HO}$ by $\mathrm{ZnPP}$ abolished IDO expression and DC maturation, while the administration resulted in opposite effects. Therefore, studying the immunosuppressive function of leukocytes in the fetomaternal interface as well as in the tumor site may help reveal the role of HO-1 in immune regulation (Blancou and Anegon, 2010; Gabrilovich et al., 2012).

\section{CONCLUSION}

HO-1 is a ubiquitous stress-response gene that is expressed and induced in a large variety of cell types. In the fetomaternal interface, HO-1 is present in several cells, such as trophoblasts (especially EVTs), endothelial cells, and infiltrating leukocytes. Accordingly, $\mathrm{HO}-1$ facilitates multiple functions during the establishment of the fetomaternal interface, which include cytoprotection, trophoblast invasion, pro-angiogenesis, and immune regulation. A deficiency of HO-1 results in pregnancy failure. Similarly, HO-1 is also expressed in malignant cells and infiltrating leukocytes in tumor sites, and shares comparable biological functions to promote tumor progression as those observed during placental development. Teasing out the function of HO-1 in each cell type may prove challenging and intriguing; but if successful, would greatly enhance our understanding of HO-1 biology as well as of the complex physiological and pathological processes of pregnancy and tumor formation. The implications of the similarities of the two systems will help in the design of integrated approaches to study pregnancy and cancer.

\section{ACKNOWLEDGMENTS}

This work was supported, in part, by the March of Dimes Prematurity Research Center at Stanford and the Stanford Child Health Research Institute, the Mary L. Johnson Research Fund, and the Christopher Hess Research Fund. 


\section{REFERENCES}

Abraham, N. G., Lin, J. H., Schwartzman, M. L., Levere, R. D., and Shibahara, S. (1988). The physiological significance of heme oxygenase. Int. J. Biochem. 20, 543-558. doi: 10.1016/0020-711X(88)90093-6

Ahmed, A., Rahman, M., Zhang, X., Acevedo, C. H., Nijjar, S., Rushton, I., et al. (2000). Induction of placental heme oxygenase-1 is protective against TNFalphainduced cytotoxicity and promotes vessel relaxation. Mol. Med. 6, 391-409.

Andersen, M. H., Sorensen, R. B., Brimnes, M. K., Svane, I. M., Becker, J. C., and thor Straten, P. (2009). Identification of heme oxygenase-1-specific regulatory CD8+ T cells in cancer patients. J. Clin. Invest. 119, 2245-2256. doi: 10.1172/JCI38739

Appleton, S. D., Marks, G. S., Nakatsu, K., Brien, J. F., Smith, G. N., and Graham, C. H. (2002). Heme oxygenase activity in placenta: direct dependence on oxygen availability. Am. J. Physiol. Heart Circ. Physiol. 282, H2055-H2059. doi: 10.1152/ajpheart.01084.2001

Appleton, S. D., Marks, G. S., Nakatsu, K., Brien, J. F., Smith, G. N., Graham, C. H., et al. (2003). Effects of hypoxia on heme oxygenase expression in human chorionic villi explants and immortalized trophoblast cells. Am. J. Physiol. Heart Circ. Physiol. 284, H853-H858. doi: 10.1152/ajpheart.00655.2002

Arck, P. C., and Hecher, K. (2013). Fetomaternal immune cross-talk and its consequences for maternal and offspring's health. Nat. Med. 19, 548-556. doi: $10.1038 / \mathrm{nm} .3160$

Arnold, J. N., Magiera, L., Kraman, M., and Fearon, D. T. (2014). Tumoral immune suppression by macrophages expressing fibroblast activation protein-alpha and heme oxygenase-1. Cancer Immunol. Res. 2, 121-126. doi: 10.1158/23266066.CIR-13-0150

Baum, M., Schiff, E., Kreiser, D., Dennery, P. A., Stevenson, D. K., Rosenthal, T., et al. (2000). End-tidal carbon monoxide measurements in women with pregnancyinduced hypertension and preeclampsia. Am. J. Obstet. Gynecol. 183, 900-903. doi: 10.1067/mob.2000.109047

Becker, T., Zu Vilsendorf, A. M., Terbish, T., Klempnauer, J., and Jorns, A. (2007). Induction of heme oxygenase-1 improves the survival of pancreas grafts by prevention of pancreatitis after transplantation. Transplantation $84,1644-1655$. doi: 10.1097/01.tp.0000290233.81395.81

Berberat, P. O., Dambrauskas, Z., Gulbinas, A., Giese, T., Giese, N., Kunzli, B., et al. (2005). Inhibition of heme oxygenase-1 increases responsiveness of pancreatic cancer cells to anticancer treatment. Clin. Cancer Res. 11, 3790-3798. doi: 10.1158/1078-0432.CCR-04-2159

Biburger, M., Theiner, G., Schadle, M., Schuler, G., and Tiegs, G. (2010). Pivotal Advance: heme oxygenase 1 expression by human CD4+ T cells is not sufficient for their development of immunoregulatory capacity. J. Leukoc. Biol. 87, 193-202. doi: 10.1189/jlb.0508280

Bilban, M., Haslinger, P., Prast, J., Klinglmuller, F., Woelfel, T., Haider, S., et al. (2009). Identification of novel trophoblast invasion-related genes: heme oxygenase-1 controls motility via peroxisome proliferator-activated receptor gamma. Endocrinology 150, 1000-1013. doi: 10.1210/en.2008-0456

Blancou, P., and Anegon, I. (2010). Editorial: heme oxygenase-1 and dendritic cells: what else? J. Leukoc Biol. 87, 185-187. doi: 10.1189/jlb.0909636

Burton, G. J., Jauniaux, E., and Watson, A. L. (1999). Maternal arterial connections to the placental intervillous space during the first trimester of human pregnancy: the Boyd collection revisited. Am. J. Obstet. Gynecol. 181, 718-724. doi: 10.1016/S0002-9378(99)70518-1

Chauveau, C., Remy, S., Royer, P. J., Hill, M., Tanguy-Royer, S., Hubert, F. X. et al. (2005). Heme oxygenase-1 expression inhibits dendritic cell maturation and proinflammatory function but conserves IL-10 expression. Blood 106, 1694-1702. doi: 10.1182/blood-2005-02-0494

Choi, A. M., and Alam, J. (1996). Heme oxygenase-1: function, regulation, and implication of a novel stress-inducible protein in oxidant-induced lung injury. Am. J. Respir. Cell Mol. Biol. 15, 9-19. doi: 10.1165/ajrcmb.15.1.8679227

Choi, K. M., Kashyap, P. C., Dutta, N., Stoltz, G. J., Ordog, T., Shea Donohue, T., et al. (2010). CD206-positive M2 macrophages that express heme oxygenase-1 protect against diabetic gastroparesis in mice. Gastroenterology 138, 2399-2409. doi: 10.1053/j.gastro.2010.02.014

Cruse, I., and Maines, M. D. (1988). Evidence suggesting that the two forms of heme oxygenase are products of different genes. J. Biol. Chem. 263, 3348-3353.

Cudmore, M., Ahmad, S., Al-Ani, B., Fujisawa, T., Coxall, H., Chudasama, K., et al. (2007). Negative regulation of soluble Flt-1 and soluble endoglin release by heme oxygenase-1. Circulation 115, 1789-1797. doi: 10.1161/CIRCULATIONAHA.106.660134
Curti, A., Trabanelli, S., Salvestrini, V., Baccarani, M., and Lemoli, R. M. (2009). The role of indoleamine 2,3-dioxygenase in the induction of immune tolerance: focus on hematology. Blood 113, 2394-2401. doi: 10.1182/blood-2008-07-144485

Deininger, M. H., Meyermann, R., Trautmann, K., Duffner, F., Grote, E. H., Wickboldt, J., etal. (2000). Heme oxygenase (HO)-1 expressing macrophages/microglial cells accumulate during oligodendroglioma progression. Brain Res. 882, 1-8. doi: 10.1016/S0006-8993(00)02594-4

Denschlag, D., Marculescu, R., Unfried, G., Hefler, L. A., Exner, M., Hashemi, A., et al. (2004). The size of a microsatellite polymorphism of the haem oxygenase 1 gene is associated with idiopathic recurrent miscarriage. Mol. Hum. Reprod. 10, 211-214. doi: 10.1093/molehr/gah024

Deramaudt, B. M., Braunstein, S., Remy, P., and Abraham, N. G. (1998). Gene transfer of human heme oxygenase into coronary endothelial cells potentially promotes angiogenesis. J. Cell. Biochem. 68, 121-127. doi: 10.1002/(SICI)10974644(19980101)68:1<121::AID-JCB12>3.0.CO;2-K

Deshane, J., Chen, S., Caballero, S., Grochot-Przeczek, A., Was, H., Li Calzi, S., et al. (2007). Stromal cell-derived factor 1 promotes angiogenesis via a heme oxygenase 1-dependent mechanism. J. Exp. Med. 204, 605-618. doi: 10.1084/jem.20061609

Doi, K., Akaike, T., Fujii, S., Tanaka, S., Ikebe, N., Beppu, T., et al. (1999). Induction of haem oxygenase-1 nitric oxide and ischaemia in experimental solid tumours and implications for tumour growth. Br. J. Cancer 80, 1945-1954. doi: 10.1038/sj.bjc.6690624

Du, M. R., Zhou, W. H., Piao, H. L., Li, M. Q., Tang, C. L., and Li, D. J. (2012). Cyclosporin A promotes crosstalk between human cytotrophoblast and decidual stromal cell through up-regulating CXCL12/CXCR4 interaction. Hum. Reprod. 27, 1955-1965. doi: 10.1093/humrep/des111

Dulak, J., Deshane, J., Jozkowicz, A., and Agarwal, A. (2008). Heme oxygenase-1 and carbon monoxide in vascular pathobiology: focus on angiogenesis. Circulation 117, 231-241. doi: 10.1161/CIRCULATIONAHA.107.698316

Erlebacher, A. (2013). Mechanisms of T cell tolerance towards the allogeneic fetus. Nat. Rev. Immunol. 13, 23-33. doi: 10.1038/nri3361

Exner, M., Minar, E., Wagner, O., and Schillinger, M. (2004). The role of heme oxygenase-1 promoter polymorphisms in human disease. Free Radic. Biol. Med. 37, 1097-1104. doi: 10.1016/j.freeradbiomed.2004.07.008

Gabrilovich, D. I., and Nagaraj, S. (2009). Myeloid-derived suppressor cells as regulators of the immune system. Nat. Rev. Immunol. 9, 162-74. doi: 10.1038/nri2506

Gabrilovich, D. I., Ostrand-Rosenberg, S., and Bronte, V. (2012). Coordinated regulation of myeloid cells by tumours. Nat. Rev. Immunol. 12, 253-268. doi: 10.1038/nri3175

George, E. M., Arany, M., Cockrell, K., Storm, M. V., Stec, D. E., and Granger, J. P. (2011a). Induction of heme oxygenase-1 attenuates sFlt-1-induced hypertension in pregnant rats. Am. J. Physiol. Regul. Integr. Comp. Physiol. 301, R1495-R1500. doi: 10.1152/ajpregu.00325.2011

George, E. M., Cockrell, K., Aranay, M., Csongradi, E., Stec, D. E., and Granger, J. P. (2011b). Induction of heme oxygenase 1 attenuates placental ischemia-induced hypertension. Hypertension 57, 941-948. doi: 10.1161/HYPERTENSIONAHA.111.169755

George, E. M., Colson, D., Dixon, J., Palei, A. C., and Granger, J. P. (2012). Heme oxygenase- 1 attenuates hypoxia-induced sFlt-1 and oxidative stress in placental villi through its metabolic products $\mathrm{CO}$ and bilirubin. Int. J. Hypertens. 2012: 486053. doi: 10.1155/2012/486053

George, J. F., Braun, A., Brusko, T. M., Joseph, R., Bolisetty, S., Wasserfall, C. H., et al. (2008). Suppression by CD4+CD25+ regulatory T cells is dependent on expression of heme oxygenase-1 in antigen-presenting cells. Am. J. Pathol. 173, 154-160. doi: 10.2353/ajpath.2008.070963

Gustafsson, C., Mjosberg, J., Matussek, A., Geffers, R., Matthiesen, L., Berg, G., et al. (2008). Gene expression profiling of human decidual macrophages: evidence for immunosuppressive phenotype. PLoS ONE 3:e2078. doi: 10.1371/journal.pone.0002078

Hanna, J., Wald, O., Goldman-Wohl, D., Prus, D., Markel, G., Gazit, R., et al. (2003). CXCL12 expression by invasive trophoblasts induces the specific migration of CD16- human natural killer cells. Blood 102, 1569-1577. doi: 10.1182/blood2003-02-0517

Holtan, S. G., Creedon, D. J., Haluska, P., and Markovic, S. N. (2009). Cancer and pregnancy: parallels in growth, invasion, and immune modulation and implications for cancer therapeutic agents. Mayo Clin. Proc. 84, 985-1000. doi: $10.1016 /$ S0025-6196(11)60669-1 
Jaffe, R., Jauniaux, E., and Hustin, J. (1997). Maternal circulation in the firsttrimester human placenta-myth or reality? Am. J. Obstet. Gynecol. 176, 695-705. doi: 10.1016/S0002-9378(97)70572-6

James, J. L., Stone, P. R., and Chamley, L. W. (2006). The regulation of trophoblast differentiation by oxygen in the first trimester of pregnancy. Hum. Reprod. Update 12, 137-144. doi: 10.1093/humupd/dmi043

Jung, I. D., Lee, J. S., Lee, C. M., Noh, K. T., Jeong, Y. I., Park, W. S., et al. (2010). Induction of indoleamine 2,3-dioxygenase expression via heme oxygenase-1-dependant pathway during murine dendritic cell maturation. Biochem. Pharmacol. 80, 491-505. doi: 10.1016/j.bcp.2010.04.025

Kovtunovych, G., Eckhaus, M. A., Ghosh, M. C., Ollivierre-Wilson, H., and Rouault, T. A. (2010). Dysfunction of the heme recycling system in heme oxygenase 1deficient mice: effects on macrophage viability and tissue iron distribution. Blood 116, 6054-6062. doi: 10.1182/blood-2010-03-272138

Kovtunovych, G., Ghosh, M. C., Ollivierre, W., Weitzel, R. P., Eckhaus, M. A., Tisdale, J. F., et al. (2014). Wild-type macrophages reverse disease in heme oxygenase 1-deficient mice. Blood 124, 1522-1530. doi: 10.1182/blood-2014-02-554162

Kreiser, D., Baum, M., Seidman, D. S., Fanaroff, A., Shah, D., Hendler, I., et al. (2004). End tidal carbon monoxide levels are lower in women with gestational hypertension and pre-eclampsia. J. Perinatol. 24, 213-217. doi: 10.1038/sj.jp.7211062

Lee, T. S., and Chau, L. Y. (2002). Heme oxygenase-1 mediates the anti-inflammatory effect of interleukin-10 in mice. Nat. Med. 8, 240-246. doi: 10.1038/nm0302-240

Li Volti, G., Sacerdoti, D., Sangras, B., Vanella, A., Mezentsev, A., Scapagnini, G., et al. (2005). Carbon monoxide signaling in promoting angiogenesis in human microvessel endothelial cells. Antioxid. Redox. Signal. 7, 704-710. doi: 10.1089/ars.2005.7.704

Lin, C. W., Shen, S. C., Hou, W. C., Yang, L. Y., and Chen, Y. C. (2008). Heme oxygenase-1 inhibits breast cancer invasion via suppressing the expression of matrix metalloproteinase-9. Mol. Cancer Ther. 7, 1195-1206. doi: 10.1158/15357163.MCT-07-2199

Linzke, N., Schumacher, A., Woidacki, K., Croy, B. A., and Zenclussen, A. C. (2014). Carbon monoxide promotes proliferation of uterine natural killer cells and remodeling of spiral arteries in pregnant hypertensive heme oxygenase-1 mutant mice. Hypertension 63, 580-588. doi: 10.1161/HYPERTENSIONAHA.113.02403

Loboda, A., Jazwa, A., Grochot-Przeczek, A., Rutkowski, A. J., Cisowski, J., Agarwal, A., et al. (2008). Heme oxygenase- 1 and the vascular bed: from molecular mechanisms to therapeutic opportunities. Antioxid. Redox. Signal. 10, 1767-1812. doi: 10.1089/ars.2008.2043

Lyall, F., Barber, A., Myatt, L., Bulmer, J. N., and Robson, S. C. (2000). Hemeoxygenase expression in human placenta and placental bed implies a role in regulation of trophoblast invasion and placental function. FASEB J. 14, 208-219.

Ma, Q., Jones, D., Borghesani, P. R., Segal, R. A., Nagasawa, T., Kishimoto, T., et al. (1998). Impaired B-lymphopoiesis, myelopoiesis, and derailed cerebellar neuron migration in CXCR4- and SDF-1-deficient mice. Proc. Natl. Acad. Sci. U.S.A. 95, 9448-9453. doi: 10.1073/pnas.95.16.9448

Maines, M. D. (1988). Heme oxygenase: function, multiplicity, regulatory mechanisms, and clinical applications. FASEB J. 2, 2557-2568.

Maines, M. D. (1997). The heme oxygenase system: a regulator of second messenger gases. Annu. Rev. Pharmacol. Toxicol. 37, 517-554. doi: 10.1146/annurev.pharmtox.37.1.517

Marks, G. S., Brien, J. F., Nakatsu, K., and McLaughlin, B. E. (1991). Does carbon monoxide have a physiological function? Trends Pharmacol. Sci. 12, 185-188. doi: 10.1016/0165-6147(91)90544-3

McCoubrey, W. K. Jr., Huang, T. J., and Maines, M. D. (1997). Isolation and characterization of a cDNA from the rat brain that encodes hemoprotein heme oxygenase-3. Eur. J. Biochem. 247, 725-732. doi: 10.1111/j.14321033.1997.00725.x

McLaughlin, B. E., Lash, G. E., Smith, G. N., Marks, G. S., Nakatsu, K., Graham, C. H., et al. (2003). Heme oxygenase expression in selected regions of term human placenta. Exp. Biol. Med. (Maywood) 228, 564-567.

McLean, M., Bowman, M., Clifton, V., Smith, R., and Grossman, A. B. (2000). Expression of the heme oxygenase-carbon monoxide signalling system in human placenta. J. Clin. Endocrinol. Metab. 85, 2345-2349. doi: 10.1210/jcem.85.6.6705

Moreau, A., Hill, M., Thebault, P., Deschamps, J. Y., Chiffoleau, E., Chauveau, C., et al. (2009). Tolerogenic dendritic cells actively inhibit $\mathrm{T}$ cells through heme oxygenase- 1 in rodents and in nonhuman primates. FASEB J. 23, 3070-3077. doi: $10.1096 /$ fj. $08-128173$
Noh, S. J., Bae, J. S., Jamiyandorj, U., Park, H. S., Kwon, K. S., Jung, S. H., et al. (2013) Expression of nerve growth factor and heme oxygenase-1 predict poor survival of breast carcinoma patients. BMC Cancer 13:516. doi: 10.1186/1471-240713-516

Park, D. J., Agarwal, A., and George, J. F. (2010). Heme oxygenase-1 expression in murine dendritic cell subpopulations: effect on CD8+ dendritic cell differentiation in vivo. Am. J. Pathol. 176, 2831-2839. doi: 10.2353/ajpath.2010.00845

Poss, K. D., and Tonegawa, S. (1997). Reduced stress defense in heme oxygenase 1-deficient cells. Proc. Natl. Acad. Sci. U.S.A. 94, 10925-10930. doi: 10.1073/pnas.94.20.10925

Ratajczak, M. Z., Zuba-Surma, E., Kucia, M., Reca, R., Wojakowski, W., and Ratajczak, J. (2006). The pleiotropic effects of the SDF-1-CXCR4 axis in organogenesis, regeneration and tumorigenesis. Leukemia 20, 1915-1924. doi: 10.1038/sj.leu. 2404357

Remy, S., Blancou, P., Tesson, L., Tardif, V., Brion, R., Royer, P. J., et al. (2009). Carbon monoxide inhibits TLR-induced dendritic cell immunogenicity. J. Immunol. 182, 1877-1884. doi: 10.4049/jimmunol.0802436

Ren, L., Liu, Y. Q., Zhou, W. H., and Zhang, Y. Z. (2012). Trophoblastderived chemokine CXCL12 promotes CXCR4 expression and invasion of human first-trimester decidual stromal cells. Hum. Reprod. 27, 366-374. doi: 10.1093/humrep/der395

Rodesch, F., Simon, P., Donner, C., and Jauniaux, E. (1992). Oxygen measurements in endometrial and trophoblastic tissues during early pregnancy. Obstet. Gynecol. 80, 283-285.

Rowe, J. H., Ertelt, J. M., Xin, L., and Way, S. S. (2012). Pregnancy imprints regulatory memory that sustains anergy to fetal antigen. Nature 490, 102-106. doi: 10.1038/nature11462

Ryter, S. W., Alam, J., and Choi, A. M. (2006). Heme oxygenase-1/carbon monoxide: from basic science to therapeutic applications. Physiol. Rev. 86, 583-650. doi: 10.1152/physrev.00011.2005

Schumacher, A., Wafula, P. O., Teles, A., El-Mousleh, T., Linzke, N., Zenclussen, M. L., et al. (2012). Blockage of heme oxygenase-1 abrogates the protective effect of regulatory T cells on murine pregnancy and promotes the maturation of dendritic cells. PLoS ONE 7:e42301. doi: 10.1371/journal.pone.002301

Shibahara, S., Nakayama, M., Kitamuro, T., Udono-Fujimori, R., and Takahashi, K. (2003). Repression of heme oxygenase-1 expression as a defense strategy in humans. Exp. Biol. Med. (Maywood) 228, 472-473.

Sierra-Filardi, E., Vega, M. A., Sanchez-Mateos, P., Corbi, A. L., and Puig-Kroger, A. (2010). Heme Oxygenase-1 expression in M-CSF-polarized M2 macrophages contributes to LPS-induced IL-10 release. Immunobiology 215, 788-795. doi: 10.1016/j.imbio.2010.05.020

Song, R., Mahidhara, R. S., Zhou, Z., Hoffman, R. A., Seol, D. W., Flavell, R. A., et al. (2004). Carbon monoxide inhibits T lymphocyte proliferation via caspase-dependent pathway. J. Immunol. 172, 1220-1226. doi: 10.4049/jimmunol.172.2.1220

Torisu-Itakura, H., Furue, M., Kuwano, M., and Ono, M. (2000). Co-expression of thymidine phosphorylase and heme oxygenase-1 in macrophages in human malignant vertical growth melanomas. Jpn. J. Cancer Res. 91, 906-910. doi: 10.1111/j.1349-7006.2000.tb01033.x

Tsai, J. R., Wang, H. M., Liu, P. L., Chen, Y. H., Yang, M. C., Chou, S. H., et al. (2012). High expression of heme oxygenase- 1 is associated with tumor invasiveness and poor clinical outcome in non-small cell lung cancer patients. Cell. Oncol. (Dordr.) 35, 461-471. doi: 10.1007/s13402-012-0105-5

Tzima, S., Victoratos, P., Kranidioti, K., Alexiou, M., and Kollias, G. (2009). Myeloid heme oxygenase-1 regulates innate immunity and autoimmunity by modulating IFN-beta production. J. Exp. Med. 206, 1167-1179. doi: 10.1084/jem. 2001582

Was, H., Cichon, T., Smolarczyk, R., Rudnicka, D., Stopa, M., Chevalier, C., et al. (2006). Overexpression of heme oxygenase-1 in murine melanoma: increased proliferation and viability of tumor cells, decreased survival of mice. Am. J. Pathol. 169, 2181-2198. doi: 10.2353/ajpath.2006.051365

Was, H., Dulak, J., and Jozkowicz, A. (2010). Heme oxygenase-1 in tumor biology and therapy. Curr. Drug Targets 11, 1551-1570. doi: $10.2174 / 1389450111009011551$

Watanabe, S., Akagi, R., Mori, M., Tsuchiya, T., and Sassa, S. (2004). Marked developmental changes in heme oxygenase-1 (HO-1) expression in the mouse placenta: correlation between HO- 1 expression and placental development. Placenta 25, 387-395. doi: 10.1016/j.placenta.2003.10.012 
Weis, N., Weigert, A., von Knethen, A., and Brune, B. (2009). Heme oxygenase-1 contributes to an alternative macrophage activation profile induced by apoptotic cell supernatants. Mol. Biol. Cell 20, 1280-1288. doi: 10.1091/mbc.E0810-1005

Welford, A. F., Biziato, D., Coffelt, S. B., Nucera, S., Fisher, M., Pucci, F., et al. (2011). TIE2-expressing macrophages limit the therapeutic efficacy of the vascular-disrupting agent combretastatin A4 phosphate in mice. J. Clin. Invest. 121, 1969-1973. doi: 10.1172/JCI44562

Wong, R. J., Zhao, H., and Stevenson, D. K. (2012). A deficiency in haem oxygenase1 induces foetal growth restriction by placental vasculature defects. Acta Paediatr. 101, 827-834. doi: 10.1111/j.1651-2227.2012.02729.x

$\mathrm{Xu}$, C., Li, J., Lu, Y., and Jiang, Z. (2012). Estrogen receptor alpha and hedgehog signal pathway developmental biology of gastric adenocarcinoma. Hepatogastroenterology 59, 1319-1322. doi: 10.5754/hge11549

Yoshiki, N., Kubota, T., and Aso, T. (2000). Expression and localization of heme oxygenase in human placental villi. Biochem. Biophys. Res. Commun. 276, 1136 1142. doi: 10.1006/bbrc.2000.3551

Zelenay, S., Chora, A., Soares, M. P., and Demengeot, J. (2007). Heme oxygenase-1 is not required for mouse regulatory $\mathrm{T}$ cell development and function. Int. Immunol. 19, 11-18. doi: 10.1093/intimm/dxl116

Zenclussen, A. C., Gerlof, K., Zenclussen, M. L., Ritschel, S., Zambon Bertoja, A., Fest, S., et al. (2006a). Regulatory T cells induce a privileged tolerant microenvironment at the fetal-maternal interface. Eur. J. Immunol. 36, 82-94. doi: 10.1002/eji.200535428

Zenclussen, M. L., Anegon, I., Bertoja, A. Z., Chauveau, C., Vogt, K., Gerlof, K. et al. (2006b). Over-expression of heme oxygenase-1 by adenoviral gene transfer improves pregnancy outcome in a murine model of abortion. J. Reprod. Immunol. 69, 35-52. doi: 10.1016/j.jri.2005.10.001

Zenclussen, A. C., Joachim, R., Hagen, E., Peiser, C., Klapp, B. F., and Arck, P. C. (2002). Heme oxygenase is downregulated in stress-triggered and interleukin-12mediated murine abortion. Scand. J. Immunol. 55, 560-569. doi: 10.1046/j.13653083.2002.01091.x
Zenclussen, A. C., Sollwedel, A., Bertoja, A. Z., Gerlof, K., Zenclussen, M. L., Woiciechowsky, C., et al. (2005). Heme oxygenase as a therapeutic target in immunological pregnancy complications. Int. Immunopharmacol. 5, 41-51. doi: 10.1016/j.intimp.2004.09.011

Zenclussen, M. L., Casalis, P. A., El-Mousleh, T., Rebelo, S., Langwisch, S., Linzke, N., et al. (2011). Haem oxygenase-1 dictates intrauterine fetal survival in mice via carbon monoxide. J. Pathol. 225, 293-304. doi: 10.1002/path.2946

Zhao, H., Azuma, J., Kalish, F., Wong, R. J., and Stevenson, D. K. (2011). Maternal heme oxygenase 1 regulates placental vasculature development via angiogenic factors in mice. Biol. Reprod. 85, 1005-1012. doi: 10.1095/biolreprod.111. 093039

Zhao, H., Wong, R. J., Kalish, F. S., Nayak, N. R., and Stevenson, D. K. (2009). Effect of heme oxygenase-1 deficiency on placental development. Placenta 30, 861-868. doi: 10.1016/j.placenta.2009.07.012

Conflict of Interest Statement: The authors declare that the research was conducted in the absence of any commercial or financial relationships that could be construed as a potential conflict of interest.

Received: 28 November 2014; paper pending published: 17 December 2014; accepted: 18 December 2014; published online: 14 January 2015.

Citation: Zhao H, Ozen M, Wong RJ and Stevenson DK (2015) Heme oxygenase-1 in pregnancy and cancer: similarities in cellular invasion, cytoprotection, angiogenesis, and immunomodulation. Front. Pharmacol. 5:295. doi: 10.3389/fphar.2014.00295

This article was submitted to Obstetric and Pediatric Pharmacology, a section of the journal Frontiers in Pharmacology.

Copyright (c) 2015 Zhao, Ozen, Wong and Stevenson. This is an open-access article distributed under the terms of the Creative Commons Attribution License (CC BY). The use, distribution or reproduction in other forums is permitted, provided the original author(s) or licensor are credited and that the original publication in this journal is cited, in accordance with accepted academic practice. No use, distribution or reproduction is permitted which does not comply with these terms. 